\title{
Laser-induced ultrasound transmitters in a flexible photoacoustic and ultrasound tomography setup
}

Gasteau, D., Thompson, D., Nagel, J., van Hespen, J. C. G., Manohar, S.

D. Gasteau, D. Thompson, J. R. Nagel, J. C. G. van Hespen, S. Manohar, "Laser-induced ultrasound transmitters in a flexible photoacoustic and ultrasound tomography setup," Proc. SPIE 11240, Photons Plus Ultrasound: Imaging and Sensing 2020, 1124044 (17 February 2020); doi: $10.1117 / 12.2544856$

SPIE. Event: SPIE BiOS, 2020, San Francisco, California, United States 


\title{
Laser-induced ultrasound transmitters in a flexible photoacoustic and ultrasound tomography setup
}

\author{
D. Gasteau ${ }^{\mathrm{a}}$, D. Thompson ${ }^{\mathrm{a}}$, J.R. Nagel ${ }^{\mathrm{a}}$, J.C.G. van Hespen ${ }^{\mathrm{b}}$, and S. Manohar ${ }^{\mathrm{a}}$ \\ ${ }^{a}$ Multi-Modality Medical Imaging Group (M3I), Technical Medical Centre, University of \\ Twente, The Netherlands \\ ${ }^{\mathrm{b}}$ Department of Biomedical Photonic Imaging, Technical Medical Centre, University of \\ Twente, The Netherlands
}

\begin{abstract}
We present a new laboratory setup for photoacoustic, transmission ultrasound and reflection ultrasound tomography. The system is based on a pair of independently rotating hemisphere segments on which are mounted acoustic transmitters and detectors. The interchangeability of the elements, as well as the ability to fully customise the acquisition protocol, allows for a considerable amount of flexibility in testing out different imaging approaches in both 2D and 3D. The current focus is the development of laser-induced ultrasound (LIUS) transmitters for use in tomographic imaging. We propose a transmitter design tuned for speed-of-sound mapping with a $1 \mathrm{MHz}$ centred response.
\end{abstract}

Keywords: Photoacoustic tomography, Laser-induced ultrasound, Speed-of-sound

\section{INTRODUCTION}

In photoacoustic (PA) imaging, reconstruction algorithms performances are highly dependent on the provided acoustic properties. In particular in breast imaging, the penetration depth of light in tissue becomes a limiting factor for the imaging depth. The deepest absorbers generate weak signal which propagates through several centimeters of inhomogeneous tissue. The main consequence of wrong acoustic properties estimation is a blurred imaged which often result in a non visible objects and a limited effective imaging depth. Accurate calculation of the PA sources locations can only be achieved using speed-of-sound (SoS) maps correctly representing the local variations of acoustic properties. ${ }^{1}$ In consequence, the development of new PA imaging systems requires to be coupled with ultrasound (US) modalities.

Combining modalities in a single experimental setup multiplies the numbers of parameters to adjust. In a PA/US setup, the choice of detectors, emitters, density of elements, illumination wavelengths, and the scanning protocol must be carefully studied in the context of the final application. Usually the acquisition of datasets as complete and dense as possible is the guarantee to improve image quality. The main drawback for clinical implementations are the longer measurement time as well as increase in both the risk of movement artifacts and burden for the patient. Moreover the time consuming processing of large amount of data may not be optimal to deliver images in practical time frames. It is important to study the influence of the sub systems in a controlled environment and optimize the imaging protocols for ideal imaging performances.

We propose a hybrid PA/US setup for tomography for 2D and 3D imaging for measurement optimization. It is aimed to be exploited as a flexible tool for optimisation of imaging approaches. In a first study, we propose to explore the use of laser-induced ultrasound (LIUS) sources as alternative to piezoelectric transducers for emission of acoustic pulses for US imaging. Typically composed of an optically absorbing material generating acoustic pulses when illuminated by a light source a LIUS source it is to be distinguished from the photoacoustic signal generated by the absorbers present in the imaged domain. It is an external and controlled ultrasound source usually fabricated from a polymer matrix with embedded absorbers such as carbon ${ }^{2}$ or gold nanoparticles. ${ }^{3,4}$ The response of the LIUS source is dependent of both optical and acoustic properties of the absorbing layer but also on the illumination choice and other aspects such as the substrate characteristics. We propose a LIUS

Further author information: (Send correspondence to D.Gasteau: E-mail: d.b.gasteau@utwente.nl

Photons Plus Ultrasound: Imaging and Sensing 2020, edited by Alexander A. Oraevsky, Lihong V. Wang,

Proc. of SPIE Vol. 11240, 1124044 · @ 2020 SPIE · CCC code: 1605-7422/20/\$21 · doi: 10.1117/12.2544856 
transmitter to be used in the newly developed hybrid imager. In contrast to previous hybrid imagers using broadband LIUS sources, ${ }^{5,6}$ the LIUS transmitter is tuned to emit $1 \mathrm{MHz}$ centred pulses for US transmission measurements of breast tissue.

\section{MATERIAL AND METHODS}

\subsection{Experimental setup}

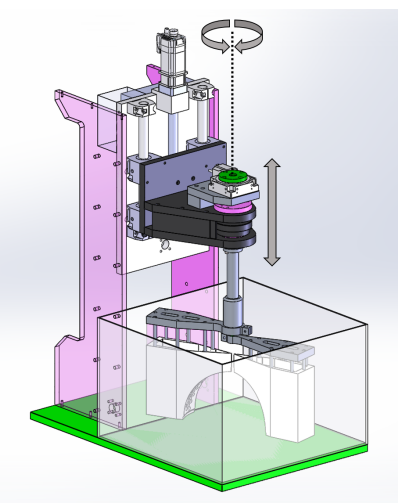

A

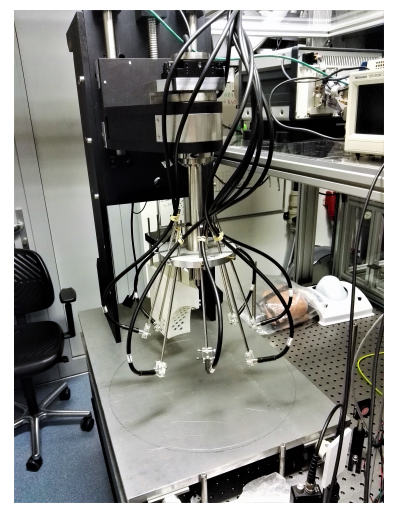

$\mathrm{B}$
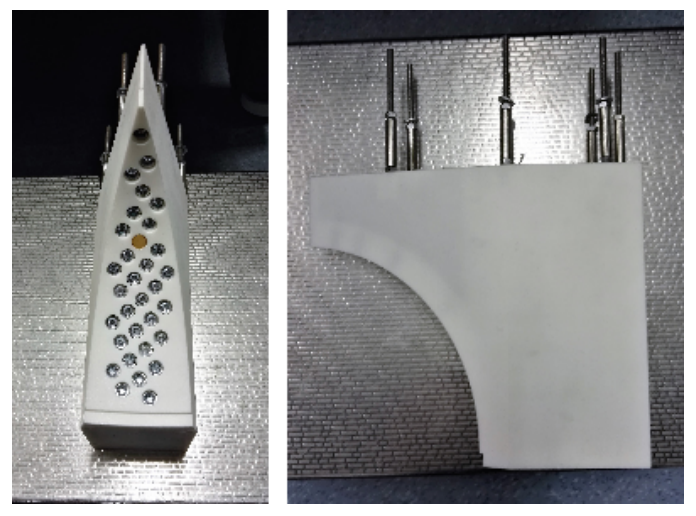

C

Figure 1. Design of the dual-modality setup showing the main moving parts (A). Photo of the setup without tank with a signal arm and fibre bundle for PA imaging. Zoom on the 3D printed nylon arms holding emitters and detectors.

The developed hybrid imaging setup presented in Fig.1 uses two suspended arms for either 2D or 3D photoacoustic and ultrasound tomographic imaging. Each arm is independently connected to their respective rotation stage granting free quasi-complete rotation around the tomography axis indicated by the vertical dashed line. Both arms are made of a 3D printed nylon structure with a curved face oriented towards the imaging domain. The curvature of the inner face is such that it mimics a hemispherical imaging domain of $13 \mathrm{~cm}$ radius. The arms are pierced in multiple places following a non-periodical spiral and in each hole can be mounted either a transducer, a LIUS transmitter or a dummy plug for unused holes. An additional translation stage allow vertical adjustment of the arms in the water tank. 3D tomographic imaging can thus be achieved by either measuring along several 2D slices or by using the multiples holes in the shaped arms in an hemispherical geometry.

Photoacoustic imaging is achieved using a single arm holding the detectors and a fibre bundle (nine outputs) for illumination. Eight fibres outputs are attached at regular angular position around the imaging domain as shown in Fig.1 B. The vertical position of each fibre can be freely adjusted whether 2D or 3D illumination is desired. In the current installation a $532 \mathrm{~nm}$ laser $10 \mathrm{~Hz}$ (Quanta Ray pro-250, Spectra Physics, USA) is coupled into the fibre bundle for photoacoustic excitation. Each fibre output is mounted with PMMA diffuser to homogenize the fluence distribution in the imaging domain. For improved illumination, the tank is enveloped by aluminium foil to reflect light escaping the tank towards the imaging domain. The recording of acoustic signal is made using highly sensitive ( $\mathrm{MDP}=0.3 \mathrm{~Pa}) 1 \mathrm{MHz}$ centred (100\% bandwidth) transducers manufactured by Imasonic (Besançon, France). The photoacoustic data is acquired by step rotation of both the arm and fibre bundle outputs around the imaging domain.

For ultrasound imaging, acoustic pulses are generated using LIUS transmitters tuned to generate $1 \mathrm{MHz}$ centred pulses with a relatively large insonification cone. The design of the LIUS transmitter is detailed further in the document. In this modality, the fibre bundle for photoacoustic illumination is removed and the two suspended arms are mounted on their respective shaft. For a 2D imaging, a single LIUS transmitter, mounted in one of the two rotating arms, is oriented in the same imaging plane as the transducer mounted in the second arm of the setup. For each position of the LIUS transmitter $L_{i}$ as shown in Fig.2, the detection arm rotates around the tomographic axis recording a partial scan at the location $D_{1}$ to $D_{M}$ as represented by the black dots. By convention, the position where emitter and detectors are facing each other is set at $0^{\circ}$. The LIUS transmitter 
acoustic beam is represented by the coloured area and the detector path is indicated by the curved double arrow with the black dots on the opposite side of the LIUS transmitter. The whole procedure is repeated by moving the two arms by given step size degrees as represented by the step A, B and C in Fig. 2 until the full scan of the phantom is achieved.

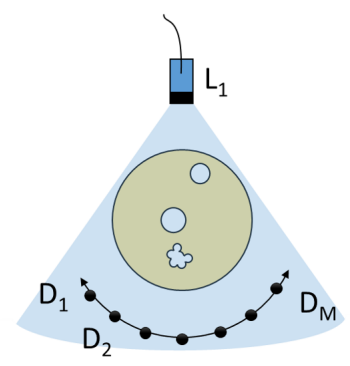

A

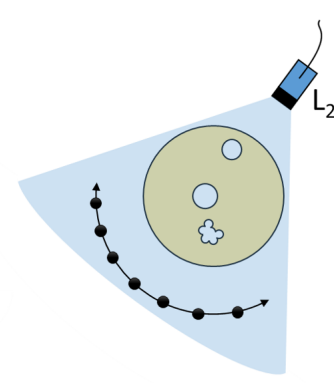

B

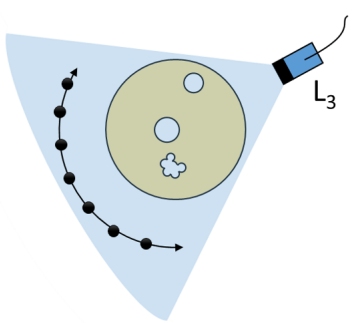

C

Figure 2. Partial scans step composing a full acoustic transmission measurement session where L indicates the LIUS transmitter position and D the detector positions.

The main advantage of this setup is the flexibility given by the straight forward assembly granting easy interchangeability of elements. Imaging performances and their dependence on experimental conditions such as detector response and acquisition protocol can be evaluated for a future use in a clinical setting. One aspect not exploited in the current work is that as the two independently rotating arms present a limited radial occupancy $\left(30^{\circ}\right)$ both reflected and transmitted ultrasound measurements can be done. However the main drawback limiting the image quality is the rotating illumination during photoacoustic imaging which means that even if diffusers help to homogenize the light distribution, the fluence distribution varies between each rotation of the detecting arms.

\subsection{LIUS transmitter}

The fabrication process of most LIUS source is based on spin coating or fibre dipping ${ }^{3,7}$ method. It usually results in thin absorbing layers generating are relatively high frequency and broadband pulses. However, for transmission imaging of larger objects such as breast tissue, acoustic waves have to propagate through several centimeters of attenuating tissue. It is then more advantageous to go towards lower acoustic frequency pulses such that acoustic attenuation and scattering have minimal impact while still remaining at a high enough frequency to obtain reasonable image resolution. In the current situation, the frequency range 0.5 to $1.5 \mathrm{MHz}$ seem to match the requirements and it allows us to use identical detectors for both photoacoustic and ultrasound modality.

As thin LIUS layers are relatively inefficient to generate low frequencies and most of the signal they generate would be undetected by low frequency transducers. The main challenge is to propose a LIUS transmitter generating acoustic pulses centered at $1 \mathrm{MHz}$. One method to achieve lower frequency is to create the absorbing layer of thickness 1 such that $\mu_{\mathrm{a}} \mathrm{l}>>1$. This means that the penetration depth is small compared to the film thickness and thus defines the frequency content of the generated pulse. Simulations using k-wave toolbox and experimental testing has shown that using a layer of $2 \mathrm{~mm}$ thick of PDMS prepared at a 10:1 ratio with $\mu_{\mathrm{a}}=6.4 \mathrm{~mm}^{-1}$ is one way to generate $1 \mathrm{MHz}$ acoustic pulses with a $1064 \mathrm{~nm}$ excitation.

The different elements necessary to use it as acoustic source are put together in a transmitter shown in Fig.3. This LIUS transmitter allows to mount an optical fibre (a) and keep it stable using the piece (b). The transmitter design is specific to allow mounting on our setup arms using threaded parts (c) and (d). The absorbing layer (f) is bonded to a spacer (e) permitting adjustment of the illumination spot size controlling the directivity of the emitted acoustic field. 


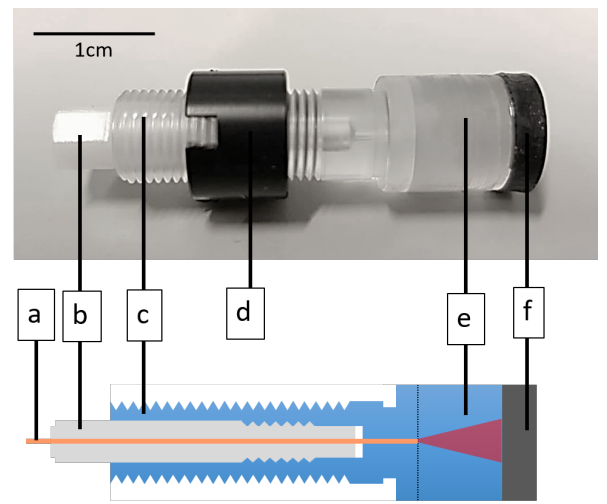

Figure 3. LIUS transmitter photo (top) and schematic (bottom). The $600 \mu \mathrm{m}$ diameter optical fibre (a) is hold in the transmitter by piece (b). Parts (c) and nut (d) allow fixation in the setup arms holes. Spacer (e) allows to adjust the illumination spot size on the absorbing layer (f) made from a PDMS and carbon black mix.

\section{RESULTS}

The acoustic response of the LIUS transmitter presented in Fig.3 has been measured on axis using a $1 \mathrm{~mm}$ diameter PVDF needle hydrophone, a submersible preamplifier and a DC coupler (Precision Acoustics, UK). The signals from this system are detected on a digitiser (DP105, Agilent Technologies, USA) built into a PC. The pressure signal shows a first bipolar transient with an rising slope following an exponential profile. It is due to the fluence profile decay from the light incidence side as expected with the Beer-Lambert law. A second pulse arriving $5 \mu \mathrm{s}$ later is identified as an internal reflection of the initial pulse propagating inside the transmitter occurring at the interface between parts (e) and (c) in Fig.3.

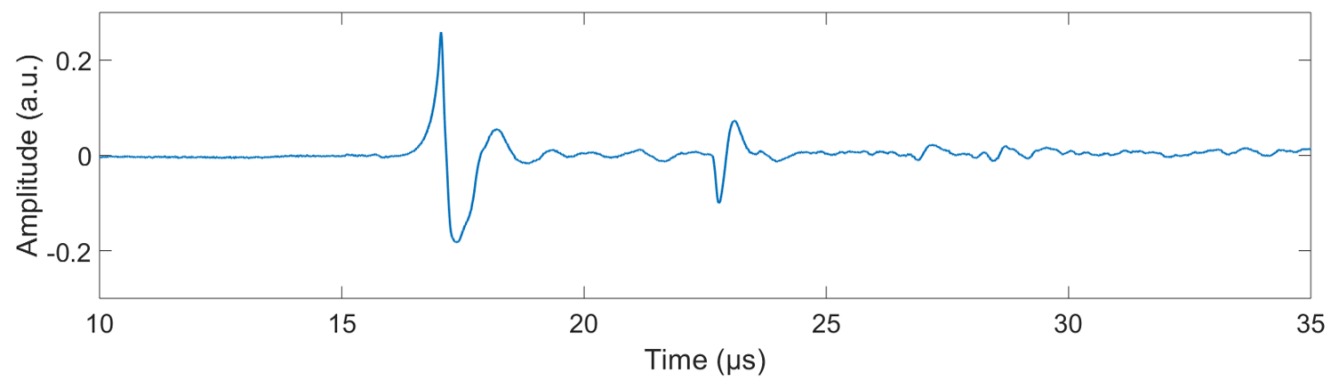

Figure 4. Pressure signal generated by the LIUS transmitter measured on axis with a 1 mm diameter PVDF hydrophone needle.

To validate the proposed transmitter design a series of preliminary scans were made in the hybrid setup with and without a speed-of-sound phantom in the imaging field. The images in Fig.5 shows two partial scans acquired following the protocol presented in Fig.2. In both case the detector is translated from $-60^{\circ}$ to $+60^{\circ}$ with a $2^{\circ}$ step size around the tomography axis. The presented results correspond to ultrasound transmission configuration. In the top image the acoustic beam is propagating undisturbed through the imaging domain. This is the measurement to be exploited as reference for time-of-flight picking. In the second case presented in the bottom image a cylindrical PVCP phantom with a measured SoS of $1395 \mathrm{~m} . \mathrm{s}^{-1}$ and water filled cavities is placed in the middle of the imaging domain. In consequence the time-of-flight is increased due to the low SoS of the phantom compared to the surrounding water. Irregular alterations are visible due to the presence of the internal cavities. 

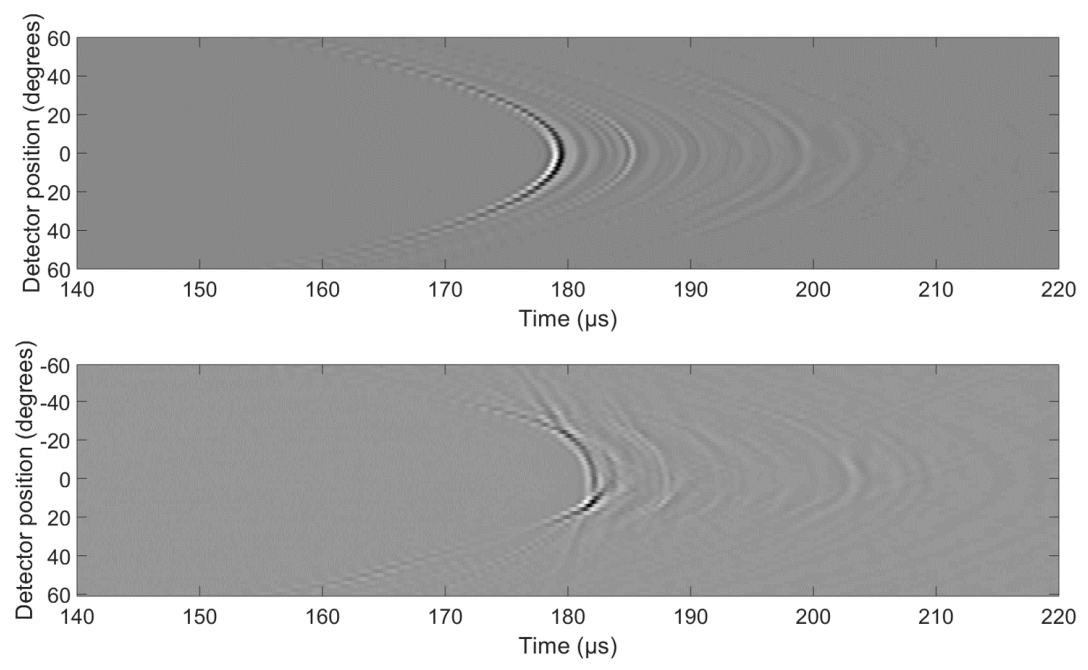

Figure 5. Partial scans measured without a phantom (top) and with a phantom (bottom)

\section{CONCLUSION}

We present a new hybrid laboratory setup to achieve photoacoustic, transmission ultrasound and reflection ultrasound tomography. The setup is develop to be easily transformable and elements modified to provide flexibility in testing out different imaging approaches in both 2D and 3D. The current interest is oriented towards the use of laser-induced ultrasound transmitters as acoustic sources for speed-of-sound evaluation. We propose a transmitter design tuned for speed-of-sound mapping with a $1 \mathrm{MHz}$ centred response optimized for transmission through breast tissue. First measurements on phantom show the expected behavior of the emitted acoustic beam distorted by the presence of a different SoS object in the imaging field.

We aim to further analyse the acquired data and obtain SoS map and compare the imaging performances of the LIUS transmitter with the results given using a conventional piezo-electric transducer. The flexible nature of the system will allow further testing of a wide range of LIUS transmitter properties, from the composition of the optically absorbing material to the generated ultrasound field profile and illumination strategy.

\section{ACKNOWLEDGMENTS}

This work was funded as a part of the European Horizon 2020 PAMMOTH project under grant agreement No 732411, an initiative of the Photonics Public-Private Partnership. The authors would like to thank Frédéric Brochin from Imasonic (Besançon, France) for providing the detectors and PA imaging (Enschede, The Netherlands) for helping with the data acquisition system.

\section{REFERENCES}

[1] Jose, J., Willemink, R. G. H., Steenbergen, W., Slump, C. H., van Leeuwen, T. G., and Manohar, S., "Speedof-sound compensated photoacoustic tomography for accurate imaging," Medical Physics 39(12), 7262-7271 (2012).

[2] Won Baac, H., Ok, J. G., Park, H. J., Ling, T., Chen, S.-L., Hart, A. J., and Guo, L. J., "Carbon nanotube composite optoacoustic transmitters for strong and high frequency ultrasound generation," Applied Physics Letters 97 (23), 234104 (2010).

[3] Noimark, S., Colchester, R. J., Poduval, R. K., Maneas, E., Alles, E. J., Zhao, T., Zhang, E. Z., Ashworth, M., Tsolaki, E., Chester, A. H., Latif, N., Bertazzo, S., David, A. L., Ourselin, S., Beard, P. C., Parkin, I. P., Papakonstantinou, I., and Desjardins, A. E., "Polydimethylsiloxane composites for optical ultrasound generation and multimodality imaging," Advanced Functional Materials 28(9), 1704919 (2018). 
[4] Hou, Y., Kim, J.-S., Ashkenazi, S., O'Donnell, M., and Guo, L. J., "Optical generation of high frequency ultrasound using two-dimensional gold nanostructure," Applied Physics Letters 89(9), 093901 (2006).

[5] Ermilov, S. A., Su, R., Conjusteau, A., Anis, F., Nadvoretskiy, V., Anastasio, M. A., and Oraevsky, A. A., "Three-dimensional optoacoustic and laser-induced ultrasound tomography system for preclinical research in mice: Design and phantom validation," Ultrasonic Imaging 38(1), 77-95 (2016). PMID: 26088582.

[6] Wurzinger, G., Nuster, R., Schmitner, N., Gratt, S., Meyer, D., and Paltauf, G., "Simultaneous threedimensional photoacoustic and laser-ultrasound tomography," Biomed. Opt. Express 4, 1380-1389 (Aug 2013).

[7] Mosse, C. A., Colchester, R. J., Bhachu, D. S., Zhang, E. Z., Papakonstantinou, I., and Desjardins, A. E., "Fiber optic ultrasound transducers with carbon/PDMS composite coatings," in [Photons Plus Ultrasound: Imaging and Sensing 2014], Oraevsky, A. A. and Wang, L. V., eds., 8943, 119 - 125, International Society for Optics and Photonics, SPIE (2014). 